\title{
An integrated electronic health record-based workflow to improve management of colonoscopy-generated pathology results
}

This article was published in the following Dove Press journal: Clinical and Experimental Gastroenterology

\author{
Spencer D Dorn \\ Holly O Cirri \\ Audrey O Chang \\ Seth D Crockett \\ Joseph A Galanko \\ John A Baron
}

Division of Gastroenterology and Hepatology, University of North Carolina School of Medicine, Chapel Hill, NC, USA
Correspondence: Spencer D Dorn Division of Gastroenterology and Hepatology, University of North Carolina at Chapel Hill, CB 7080, Chapel Hill, NC 27599-7080, USA

Email sdorn@med.unc.edu
Purpose: Managing and communicating colonoscopy-generated pathology results and appropriate follow-up recommendations can be challenging. To improve this process, we developed and implemented a standardized electronic health record-based intervention with built-in decision support.

Methods: Fourteen attending endoscopists performed enough colonoscopies to qualify for the study. For each, we randomly sampled and abstracted data from 35 colonoscopies that met prespecified inclusion criteria during both the pre-intervention and also post-intervention periods. Follow-up recommendations were compared to guidelines. We used the Wilcoxon Signed Rank Test to assess the change in the proportion of cases with guideline-concordant results, the proportion with a documented follow-up result letter, and the median time to letter completion. A brief survey assessed endoscopists' satisfaction with the intervention.

Results: In total, 1,947 colonoscopies were extracted, of which 968 met inclusion criteria. The proportion of follow-up recommendations that were guideline concordant increased from a median of $82.9 \%$ pre-intervention to $85.7 \%$ post-intervention $(P=0.72)$. The proportion of observations with a documented follow-up result letter increased from a median of $88.9 \%$ preintervention to $97.1 \%$ post-intervention $(P=0.07)$. The number of calendar days between the date of the colonoscopy and the date the letter was sent decreased from a median of 7.7 days pre-intervention to 6.8 days post-intervention $(P=0.79)$. Eighty-six percentage of endoscopists were either "very satisfied" or "satisfied" with the overall process.

Conclusion: The intervention was not associated with a statistically significant increase in guideline-concordant recommendations or efficiency measures, perhaps due to high baseline performance. The intervention was well received by endoscopists and captured data necessary for important downstream processes.

Keywords: colonoscopy, pathology, quality, electronic health record, systems

\section{Introduction}

Colonoscopy is a cost-effective ${ }^{1,2}$ colorectal cancer screening modality recommended by multiple governmental agencies ${ }^{3}$ and professional societies. ${ }^{4}$ In $30 \%-50 \%$ of colonoscopies, specimens are obtained for pathologic analysis. ${ }^{5}$ Ideally, when endoscopists receive these results (typically several days after the colonoscopy was performed) they should determine appropriate follow-up recommendations, and then clearly communicate this information to their patients and referring providers. ${ }^{5}$ In actuality, follow-up recommendations often stray from established guidelines ${ }^{6}$ and are not communicated clearly or in a timely fashion. ${ }^{7}$ Consequently, there is evidence that colonoscopy is 
overused by individuals at low risk of colorectal cancer ${ }^{8,9}$ and underused by those at high risk. ${ }^{8}$

In April 2015, we identified four problems with how endoscopists managed and communicated pathology results and follow-up recommendations. First, there was extreme variability in terms of whether, how, and when results and follow-up recommendations were communicated to patients and their referring providers. Second, follow-up recommendations often strayed from practice guidelines and prescribed surveillance intervals varied among providers. Third, the timing of the repeat colonoscopy was not reliably updated in our appointment recall system. Also, the pathology result was not captured in the discrete format needed to calculate endoscopists' adenoma detection rates (ADRs), a measure of colonoscopy quality.

\section{Methods}

In response to these deficiencies, we developed and subsequently implemented the standardized workflow that is shown in Figure 1. After completing a colonoscopy, the endoscopist generates a report that includes procedure indications, findings (including number, morphology, and size of polyps), and initial recommendations. Once finalized, this report flows as a free-text file to the EPIC (EPIC Systems, Verona, WI, USA) electronic health record (EHR). Several days later, the pathology report returns to the endoscopist's EPIC In Basket. The endoscopist reviews the pathology report and associated colonoscopy report, then generates a letter to the patient and referring physician (Figure 2). This letter is a "SmartText" that generates text based on responses to "SmartLists" that are nested within one another. The first SmartList prompts for the type of test (colonoscopy, upper endoscopy, or others). Choos- ing "colonoscopy" triggers a second SmartList that asks for the most advanced pathology category (normal, hyperplastic polyps, $1-2$ tubular adenomas $<10 \mathrm{~mm},>3$ tubular adenomas $<10 \mathrm{~mm}$, advanced adenoma, serrated lesion, sessile lesion and adenoma, or colitis). The item selected (eg, 1-2 tubular adenomas) triggers a third SmartList prompting for the patient's baseline risk (eg, personal history, family history and age). Finally, this selection triggers a fourth SmartList that prompts for a recommended timeframe for the next colonoscopy (1-10 years, none, or based on patient and referring physician preference). Importantly, this final SmartList automatically suggests a guideline-appropriate interval ${ }^{10}$ that the endoscopist can either accept or override. The endoscopist then reviews and, if necessary, edits the letter, adds signature, and clicks submit. The letter then routes to an administrative assistant who transcribes the pathology category and recall interval into the ProVation endowriter (ProVation Medical, Minneapolis, MN, USA) GI Quality Improvement Consortium (GIQuIC) form, thereby updating the GIQuIC Quality Registry and ProVation's appointment recall module. The administrative assistant then clicks "accept," automatically sending the letter to the patient and referring physician via EPIC, fax, or mail (depending on their communication preferences). The finalized letter is documented in EPIC's Chart Review.

The end result is that patients and referring physicians receive a letter that includes decision support-aided follow-up recommendations, the letter is documented in the EHR, the colonoscopy recall interval is updated in the patient appointment reminder system, and a discrete pathology category needed to calculate ADR is captured. After a 1-month learning period, we required that all endoscopists use this process to follow-up colonoscopy pathology results.

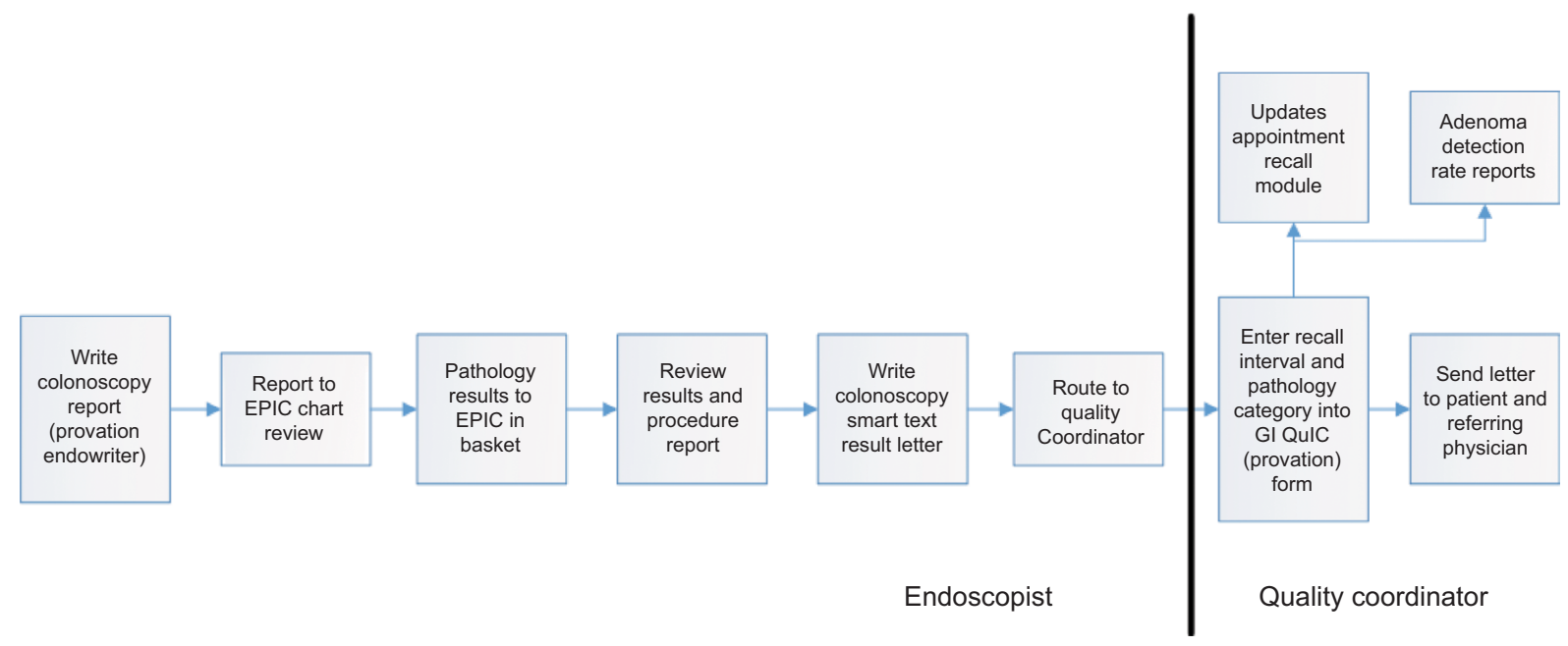

Figure I Standardized colonoscopy pathology processing workflow. 


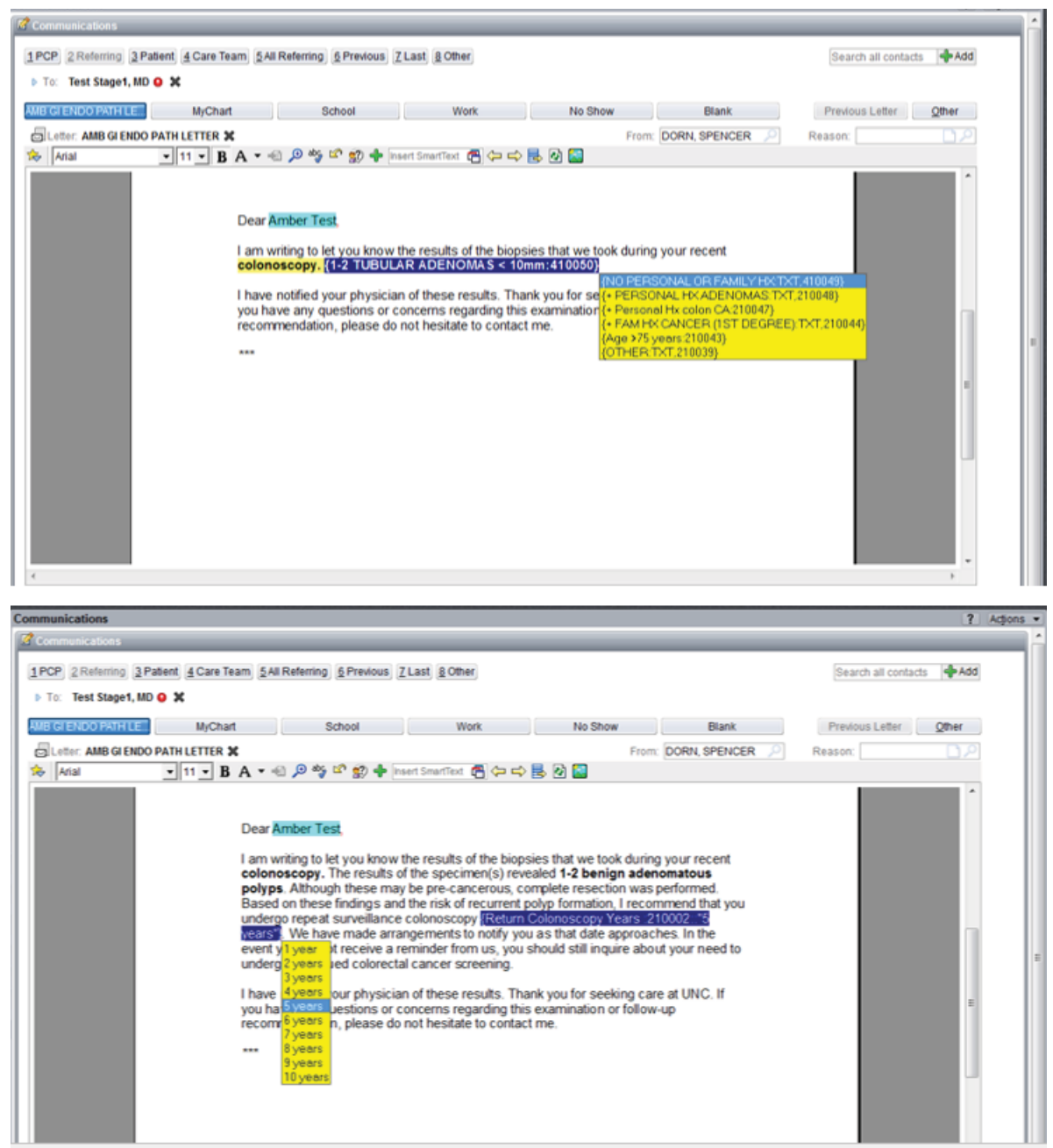

Figure 2 Colonoscopy pathology SmartText letter.

We assessed the effects of this intervention using a beforevs-after study design, as well as a brief endoscopist survey. The primary outcome was the proportion of guideline-concordant follow-up recommendations. Secondary outcomes included measures of efficiency, including the proportion of colonoscopies with a documented follow-up letter, the number of days between the colonoscopy and the letter being sent, and overall endoscopists' satisfaction with the new process.

\section{Ethical approval}

This study was approved by the University of North Carolina Institutional Review Board, who waived informed consent requirements for patients because they were not directly involved in the research study (only their already collected clinical data were used). Patient privacy and confidentiality was maintained by adhering to Good Clinical Practice and also by not abstracting any patient identifiers from the medical record. Informed consent was obtained from physician subjects who completed the survey.

\section{Study period}

The pre-intervention period was February 1 to October 15, 2015. We excluded colonoscopies performed during the soft rollout when the intervention was introduced but not mandated (October 15 to November 30, 2015). The postintervention period was December 1, 2015, to August 15, 2016.

\section{Endoscopist inclusion criteria}

We included attending gastroenterology endoscopists who performed more than 100 colonoscopies that resulted in pathology specimens that met study criteria (see below) during each of the pre-intervention and post-intervention study periods. After exclusion of two endoscopists who mostly 
perform colonoscopies in patients with inflammatory bowel disease (see exclusion criteria below), we ultimately included 14 endoscopists.

\section{Power calculation}

For the primary outcome, we estimated $75 \%$ guideline concordance during the pre-intervention period and $85 \%$ during the post-intervention period. With 14 endoscopists, we required 35 observations per endoscopist during the preintervention period and 35 observations per endoscopist during the post-intervention period (70 total) to achieve $90 \%$ power to detect a difference in outcomes between the two periods.

\section{Chart review and data abstraction}

We queried our institution's data warehouse to identify patients who underwent a screening or surveillance colonoscopy with biopsy/polypectomy at our institution performed by any faculty endoscopist who met the inclusion criteria. Colonoscopies that involved assistance from gastroenterology physician fellows were assigned to the gastroenterology attending physician who was responsible for the procedure. This is consistent with our practice's policy that attending physicians are responsible for reviewing and communicating pathology results. For each physician we generated a list of patients who underwent colonoscopies with associated pathology results during the pre-intervention period, and a second list of patients who underwent colonoscopies with associated pathology results during the post-intervention period for a total of 28 lists. For each endoscopist we randomly sampled 35 observations during the pre-intervention period and 35 observations during the post-intervention period. We excluded colonoscopies with the following characteristics: fair or poor bowel prep; diagnosis of cancer; no polypectomy; incomplete removal or retrieval of one or more polyps; incomplete examination (ie, failure to reach the cecum or surgical anastomosis); surgical recommendation; indication for diagnostic purposes (eg, diarrhea, rectal bleeding, and abdominal pain) or for colorectal surveillance in the setting of inflammatory bowel disease or a familial cancer syndrome; and patient aged $\geq 75$ years at the time of the colonoscopy. We continued the random sample process until we identified 35 pre- and 35 post-intervention observations for each endoscopist.

\section{Guideline concordance determination}

For each identified colonoscopy we abstracted the following data: endoscopist name, procedure date, patient age, colonoscopy indication, colonoscopy preparation quality (excellent, good, fair, poor), number of polyps, size of the largest polyp, most advanced polyp histology, whether the colonoscopy was completed, whether any polyps were incompletely removed or retrieved, and whether the colonoscopy recommendation included surgery. Additionally, we abstracted whether there was a documented follow-up letter in EPIC and, if so, the date of the letter and the recommended follow-up interval (ie, timing of next colonoscopy). In cases without any documented follow-up letter, we recorded the follow-up interval that was documented in the colonoscopy report. Finally, we compared the follow-up interval recommended by the endoscopist in their letter (or, if no letter, the colonoscopy report) to US Multi-Society Task Force on Colorectal Cancer guidelines, which were the most recent available at the time the intervention was designed..$^{10}$ The Multi-Society Task Force guidelines do not specifically address follow-up for patients with a family history of colon cancer, for patients with normal histopathology results, or those with hyperplastic polyps and a family history of colon cancer. In these cases, we referred to recommendations from the American College of Gastroenterology. ${ }^{11}$ Furthermore, because the Multi-Society Task Force guidelines do not provide guidance on all types of serrated lesions, for patients with serrated lesions we referred to consensus recommendations from an expert panel. ${ }^{12}$ Finally, for patients with a prior personal history of colorectal cancer we referred to 2006 guidelines from the American Cancer Society and the US Multi-Society Task force on Colorectal Cancer. ${ }^{13}$ For all cases for whom endoscopist recommendations were discordant with guidelines, we determined whether there was a reason that justified a sooner or longer interval (eg, poor patient health status or age $>75$ years).

\section{Endoscopist survey}

We administered a brief web-based survey to all endoscopists to assess the usability of the SmartText letter and their satisfaction with the overall workflow.

\section{Statistical analyses}

For each endoscopist, we assessed the change in the proportion of guideline-concordant recommendations by subtracting the pre-intervention concordance from the post-intervention concordance. The Wilcoxon Signed Rank Test was used on the change in concordance numbers to determine whether the change was significantly different from zero. For secondary outcomes we used the Wilcoxon Signed Rank test to assess the change in the proportion of cases with a documented follow-up letter, as well as the change in the median time to letter completion. Finally, descriptive statistics were used 
to assess endoscopists' self-reported satisfaction with the process.

\section{Results}

A total of 1,947 colonoscopies with associated pathology were extracted, of which 968 (49.7\%) met inclusion criteria. Eighty-nine (4.6\% of total) were excluded for inadequate bowel preparation, $108(5.6 \%)$ for incomplete polyp resection or retrieval, $4(0.2 \%)$ for incomplete examination, $478(24.6 \%)$ for diagnostic indication, 157 (8.1\%) for inflammatory bowel disease or familial cancer syndrome surveillance, $120(6.2 \%)$ for age $>75$ years, $3(0.2 \%)$ for colorectal cancer diagnosis, and $18(0.9 \%)$ for other reasons. Of the 14 endoscopists, 12 had 35 qualifying pre-intervention observations and 35 qualifying post-intervention observations. One endoscopist had only 32 qualifying pre-intervention observations and 35 qualifying post-intervention observations. Another endoscopist had 35 qualifying pre-intervention observations, but only 26 qualifying post-intervention observations. These endoscopists were still included in the analyses.

The main results are shown in Table 1 . The percentage of follow-up recommendations that were guideline concordant increased from a median of $82.9 \%$ pre-intervention to $85.7 \%$ post-intervention $(P=0.72)$. The percentage of guideline concordant recommendations for each endoscopist in the pre- and post-intervention periods is shown in Figure 3. The proportion of observations with a documented follow-up result letter increased from a median of $88.9 \%$ pre-intervention to $97.1 \%$ post-intervention $(P=0.07)$. Likewise, the number of calendar days between the date of the colonoscopy and the date the letter was sent decreased from a median of 7.7 days pre-intervention to 6.8 days post-intervention $(P=0.79)$.

Finally, of the 14 endoscopists who were included in the study, 5 (36\%) were "very satisfied" with the intervention, 7 (50\%) were "satisfied", 2 (14\%) were neither satisfied nor dissatisfied, and none were "dissatisfied" or "very dissatisfied". Self-reported time spent per result ranged from 20 seconds to 5 minutes. Self-reported time spent on all results

Table I Outcomes: pre- vs post-intervention

\begin{tabular}{|c|c|c|c|}
\hline & $\begin{array}{l}\text { Pre- } \\
\text { intervention }\end{array}$ & $\begin{array}{l}\text { Post- } \\
\text { intervention }\end{array}$ & $P$-value \\
\hline $\begin{array}{l}\text { Median \% guideline } \\
\text { concordance }\end{array}$ & $82.9 \%$ & $85.7 \%$ & 0.72 \\
\hline $\begin{array}{l}\text { Median \% with documented } \\
\text { result letter }\end{array}$ & $88.9 \%$ & $97.1 \%$ & 0.07 \\
\hline $\begin{array}{l}\text { Median calendar days } \\
\text { elapsed }\end{array}$ & 7.7 & 6.8 & 0.79 \\
\hline
\end{tabular}

each week ranged from 10 to 120 minutes. Thirteen of the $14(93 \%)$ endoscopists found ADR feedback to be useful. The main feedback for improving the system was to add additional upper endoscopy options and create a Spanish language version.

\section{Discussion}

We designed a new EHR-based process for reviewing and communicating colonoscopy pathology results with the goals of 1) improving follow-up recommendation decision making; 2) improving efficiency and physician satisfaction; and 3 ) capturing information necessary to measure quality and ensure accurate patient appointment recalls. Our findings suggest that the process achieved the latter two goals.

The percentage of patients who received guidelineconcordant follow-up recommendations was not significantly different pre- vs post-intervention. This runs counter to findings from an earlier systematic review that EHR-based decision supports embedded into the clinician workflow to be available at the time of decision making are generally effective. ${ }^{14}$ However, a more recent systematic review found that embedding decision support at the point of decision making does not predict success, perhaps due to alert fatigue. Requiring clinicians to give a reason for overriding advise can increase decision support effectiveness, though this was not a feature of our intervention. ${ }^{15}$

Relatively high guideline-concordance rates (83\%) at baseline may explain why rates did not significantly increase. Guidelines are recommendations, not rules. Guideline-based recommendations may not apply to or be incorrect for certain clinical scenarios. ${ }^{16}$ In such circumstances, clinicians should rely on their own judgment and experience. It is therefore unreasonable to expect that even close to $100 \%$ of

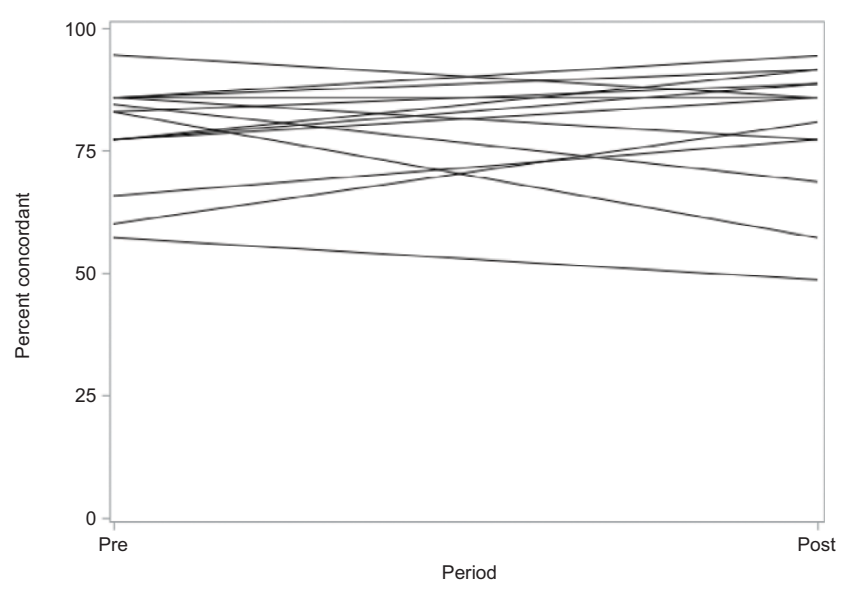

Figure 3 Percentage of guideline-concordant follow-up recommendations by individual endoscopists in the pre- and post-intervention periods. 
colonoscopy follow-up recommendations will concur with guidelines.

The intervention was associated with clinically meaningful, though not statistically significant, increases in efficiency, though the study may have been underpowered to detect changes in these secondary outcome measures. The proportion of patients who received a follow-up result letter increased to $97 \%$. It is unlikely that any process could increase this any further. In addition, post-intervention letters were written and mailed slightly sooner, on average, within 1 week of the colonoscopy. Given that pathology results are typically not available for 3-4 days, accounting for holidays and weekends, and recognizing that physicians often prefer to batch their work, we are not sure that any process could speed this up much more.

The intervention was well received by endoscopists, who were almost universally satisfied with the EHR-based process. Their main recommendations were to expand the letters to better encompass upper gastrointestinal pathology and include Spanish language, two enhancements that we recently implemented. In addition, for the first time, our practice captured polyp histopathology category in a way that allowed us to calculate ADRs, far and away the strongest gastroenterology quality measure. Furthermore, the process ensured that patient recall appointment intervals were ppropriately updated, which should reduce the risk patients will show up too early or too late for a repeat colonoscopy.

This study had several limitations. First, because this was not a randomized controlled trial, unmeasured or uncontrolled factors may have affected the study results. Second, at times it was difficult to determine whether colonoscopy follow-up recommendations were or were not guideline concordant. For instance, in one observation five small polyps were resected and placed into a single pathology specimen jar. The pathologist interpreted the specimens as "adenoma fragments" and "hyperplastic polyp fragments", making it difficult to determine whether guideline-based follow-up would be $5-10$ years, 5 years, or 3 years. Cases like this one were adjudicated by a gastroenterologist investigator who was blind to pre- vs post-intervention status and generally gave the endoscopist the "benefit of the doubt" if there were any justifiable reasons to assume that their recommendations were guideline concordant. Any nondifferential misclassification would bias the results toward the null hypothesis. Third, the number of observations was slightly unbalanced in that one endoscopist had only 32 qualifying pre-intervention observa- tions, and one had only 26 qualifying post-intervention observations. This may have reduced statistical power somewhat.

\section{Conclusion}

We developed and implemented an EHR-based process designed to improve the colonoscopy pathology result follow-up process. The intervention was not associated with a statistically significant increase in guideline-concordant recommendations or efficiency measures, perhaps due to high baseline performance and/or a result of the study being underpowered. However, it was well received by endoscopists and captured data necessary for important downstream processes. The intervention can be shared with other gastroenterology practices using the EPIC EHR through EPIC's community library, or modified for and programmed into other EHRs that have a similar letter writing functionality.

\section{Disclosure}

The authors report no conflicts of interest in this work.

\section{References}

1. Pignone M, Saha S, Hoerger T, Mandelblatt J. Cost-effectiveness analyses of colorectal cancer screening: a systematic review for the U.S. Preventive Services Task Force. Ann Intern Med. 2002;137(2): 96-104.

2. Song K, Fendrick AM, Ladabaum U. Fecal DNA testing compared with conventional colorectal cancer screening methods: a decision analysis. Gastroenterology. 2004;126(5):1270-1279.

3. US Preventive Services Task Force, Bibbins-Domingo K, Grossman DC, et al. Screening for colorectal cancer: US Preventive Services Task Force Recommendation Statement. JAMA. 2016;315(23): 2564-2575.

4. Rex DK, Boland CR, Dominitz JA, et al. Colorectal cancer screening: recommendations for physicians and patients from the U.S. Multi-Society Task Force on Colorectal Cancer. Gastroenterology. 2017;153(1):307-323.

5. Lieberman D, Nadel M, Smith RA, et al. Standardized colonoscopy reporting and data system: report of the Quality Assurance Task Group of the National Colorectal Cancer Roundtable. Gastrointest Endosc. 2007;65(6):757-766.

6. Mysliwiec PA, Brown ML, Klabunde CN, Ransohoff DF. Are physicians doing too much colonoscopy? A national survey of colorectal surveillance after polypectomy. Ann Intern Med. 2004;141(4): 264-271.

7. Fletcher RH, Nadel MR, Allen JI, et al. The quality of colonoscopy services-responsibilities of referring clinicians: a consensus statement of the Quality Assurance Task Group, National Colorectal Cancer Roundtable. J Gen Intern Med. 2010;25(11):1230-1234.

8. Lieberman D. Progress and challenges in colorectal cancer screening and surveillance. Gastroenterology. 2010;138(6):2115-2126.

9. Ransohoff DF, Yankaskas B, Gizlice Z, Gangarosa L. Recommendations for post-polypectomy surveillance in community practice. $\mathrm{Dig} \mathrm{Dis} \mathrm{Sci}$. 2011;56(9):2623-2630.

10. Lieberman DA, Rex DK, Winawer SJ, et al. Guidelines for colonoscopy surveillance after screening and polypectomy: a consensus update by the US Multi-Society Task Force on Colorectal Cancer. Gastroenterology. 2012;143(3):844-857. 
11. Rex DK, Johnson DA, Anderson JC, et al. American College of Gastroenterology guidelines for colorectal cancer screening 2009 [corrected]. Am J Gastroenterol. 2009;104(3):739-750.

12. Rex DK, Ahnen DJ, Baron JA, et al. Serrated lesions of the colorectum review and recommendations from an expert panel. Am J Gastroenterol. 2012;107(9):1315-1329.

13. Rex DK, Kahi CJ, Levin B, et al. Guidelines for colonoscopy surveillance after cancer resection: a consensus update by the American Cancer Society and the US Multi-Society Task Force on Colorectal Cancer. Gastroenterology. 2006;130(6):1865-1871.
14. Kawamoto K, Houlihan CA, Balas EA, Lobach DF. Improving clinical practice using clinical decision support systems: a systematic review of trials to identify features critical to success. $\mathrm{Br} \mathrm{Med} J$. 2005;330(7494):765.

15. Roshanov PS, Fernandes N, Wilczynski JM, et al. Features of effective computerised clinical decision support systems: meta-regression of 162 randomised trials. Br Med J. 2013;346:f657.

16. Woolf SH, Grol R, Hutchinson A, Eccles M, Grimshaw J. Clinical guidelines: potential benefits, limitations, and harms of clinical guidelines. Br Med J. 1999;318(7182):527-530.
Clinical and Experimental Gastroenterology

\section{Publish your work in this journal}

Clinical and Experimental Gastroenterology is an international, peerreviewed, open access, online journal publishing original research, reports, editorials, reviews and commentaries on all aspects of gastroenterology in the clinic and laboratory. This journal is included on PubMed. The manuscript management system is completely online

\section{Dovepress}

and includes a very quick and fair peer-review system, which is all easy to use. Visit http://www.dovepress.com/testimonials.php to read real quotes from published authors. 